Proceedings of the

International Geometry Center

Vol. 14, no. 3 (2021) pp. 206-218

\title{
On symmetry reduction and some classes of invariant solutions of the $(1+3)$-dimensional homogeneous Monge-Ampère equation
}

\author{
Vasyl Fedorchuk, Volodymyr Fedorchuk
}

\begin{abstract}
We study the relationship between structural properties of the two-dimensional nonconjugate subalgebras of the same rank of the Lie algebra of the Poincare group $P(1,4)$ and the properties of reduced equations for the $(1+3)$-dimensional homogeneous Monge-Ampère equation. In this paper, we present some of the results obtained concerning symmetry reduction of the equation under investigation to identities. Some classes of the invariant solutions (with arbitrary smooth functions) are presented.
\end{abstract}

\begin{abstract}
Анотація. Вивчається взаємозв'язок між структурними властивостями двовимірних неспряжених підалгебр того самого рангу алгебри Лі групи Пуанкаре $P(1,4)$ і властивостями редукованих рівнянь для $(1+3)$-вимірного однорідного рівняння Монжа-Ампера. У цій роботі представлено деякі отримані результати, що стосуються симетрійної редукції досліджуваного рівняння до тотожностей. Наведено деякі класи інваріантних розвязків (з довільними гладкими функціями).
\end{abstract}

2010 Mathematics Subject Classification: 22E70, 35B06, 53A07

UDC $512.813+517.957 .6$

Keywords: symmetry reduction, invariant solutions, Monge-Ampère equation, classification of Lie algebras, nonconjugate subalgebras, Poincaré group $P(1,4)$

Ключові слова: симетрійна редукція, інваріантні розв'язки, рівняння МонжаАмпера, класифікація алгебр, некомутативні підалгебри, група Пуанкаре $P(1,4)$

DOI: http://dx.doi.org/10.15673/tmgc.v14i3.2078 


\section{INTRODUCTION}

In many cases mathematical models of the processes of real world can be written with the help of partial differential equations (PDEs). Among of these equations there are a lot of PDEs with non trivial symmetry groups. To investigate of this type equations we can use the classical LieOvsiannikov method [27,31,30,29] (see, also, the references therein). The application of this approach allow us, in particular, to perform the symmetry reductions of the equations under study and to construct classes of exact solutions.

However, it turned out that in order to efficiently apply the classical LieOvsiannikov method for PDEs with non-trivial symmetry groups we had to solve a pure algebraic problem of describing all nonconjugate (nonsimilar) subalgebras of the Lie algebras of symmetry groups of the equations under investigation. More details on this theme can be found in $[31,30]$ (see, also, the references therein).

In 1975, Patera, Winternitz, and Zassenhaus [32] proposed a general method for describing the nonconjugate subalgebras of Lie algebras with nontrivial ideals.

In 1984, Grundland, Harnad, and Winternitz [20] pointed out that the reduced equations, obtained with the help of nonconjugate subalgebras of the same ranks of the Lie algebras of the symmetry groups of some PDEs, were of different types. They also investigated the similar phenomenon. The confirmation of this conclusion can be found in $[7,5,6,28,9,19,10-14]$ (see, also, the references therein).

The results obtained cannot be explained using only the rank of nonconjugate subalgebras of the Lie algebras of the symmetry groups of PDEs under investigation.

To try to explain some of the differences in the properties of the reduced equations for PDEs with nontrivial symmetry groups, we recently suggested to investigate the relationship between the structural properties of nonconjugate subalgebras of the same rank of the Lie algebras of the symmetry groups of those PDEs and the properties of the corresponding reduced equations [10]. To realise of this suggestion we need to solve pure algebraic problem of classifying of all nonconjugate subalgebras of the Lie algebras of symmetry groups of the equations under investigation into classes of isomorphic ones.

A solution a lot of problems of the geometry, string theory, geometrical optics, elastic theories of shallow shells, optimal transportation, onedimensional gas dynamics, meteorology and oceanography, etc. has reduced to the investigation of the Monge-Ampère equations in the spaces of different dimensions and different types. Some details on this theme can 
be found in $[33,1,34,37,38,24,21,26,39,22,2,15,40,25,36,23,35]$ (see, also, the references therein).

This paper is devoted to the study the relationship between structural properties of the low-dimensional $(\operatorname{dim} L \leqslant 3$ ) nonconjugate subalgebras of the same rank of the Lie algebra of the Poincare group $P(1,4)$ and the properties of reduced equations for the $(1+3)$-dimensional homogeneous Monge-Ampère equation.

At the present time, the relationship has been investigated between the structural properties of the two-dimensional nonconjugate subalgebras of the same rank of the Lie algebra of the Poincaré group $P(1,4)$ and the properties of reduced equations for the $(1+3)$-dimensional homogeneous Monge-Ampère equation.

We have obtained the following types of the reduced equations:

- identities,

- partial differential equations.

In this paper, we plan to present some of our results, which are obtained on the way of symmetry reduction of the $(1+3)$-dimensional homogeneous Monge-Ampère equation to identities.

\section{Lie algebra of the Poincaré group P(1,4) And its NONCONJUGATE SUBALGEBRAS}

The group $P(1,4)$ is a group of rotations and translations of the fivedimensional Minkowski space $M(1,4)$. It is the smallest group, which contains, as subgroups, the extended Galilei group $\widetilde{G}(1,3)[18]$ (the symmetry group of classical physics) and the Poincaré group $P(1,3)$ (the symmetry group of relativistic physics).

Lie algebra of the group $P(1,4)$ is generated by 15 bases elements

$$
M_{\mu \nu}=-M_{\nu \mu},(\mu, \nu=0,1,2,3,4), \quad P_{\mu},(\mu=0,1,2,3,4),
$$

which satisfy the commutation relations:

$$
\begin{gathered}
{\left[P_{\mu}, P_{\nu}\right]=0, \quad\left[M_{\mu \nu}, P_{\sigma}\right]=g_{\nu \sigma} P_{\mu}-g_{\mu \sigma} P_{\nu},} \\
{\left[M_{\mu \nu}, M_{\rho \sigma}\right]=g_{\mu \sigma} M_{\nu \rho}+g_{\nu \rho} M_{\mu \sigma}-g_{\mu \rho} M_{\nu \sigma}-g_{\nu \sigma} M_{\mu \rho},}
\end{gathered}
$$

where $g_{00}=-g_{11}=-g_{22}=-g_{33}=-g_{44}=1, g_{\mu \nu}=0$, if $\mu \neq \nu$.

In this paper, we consider the following representation [16] of the Lie algebra of the group $P(1,4)$ :

$$
\begin{gathered}
P_{0}=\frac{\partial}{\partial x_{0}}, \quad P_{1}=-\frac{\partial}{\partial x_{1}}, \quad P_{2}=-\frac{\partial}{\partial x_{2}}, \quad P_{3}=-\frac{\partial}{\partial x_{3}}, \\
P_{4}=-\frac{\partial}{\partial u}, \quad M_{\mu \nu}=x_{\mu} P_{\nu}-x_{\nu} P_{\mu}, x_{4} \equiv u .
\end{gathered}
$$


In what follows, we will use the next bases elements:

$$
\begin{gathered}
G=M_{04}, \quad L_{1}=M_{23}, \quad L_{2}=-M_{13}, \quad L_{3}=M_{12}, \\
P_{a}=M_{a 4}-M_{0 a}, \quad C_{a}=M_{a 4}+M_{0 a}, \quad(a=1,2,3), \\
X_{0}=\frac{1}{2}\left(P_{0}-P_{4}\right), \quad X_{k}=P_{k}(k=1,2,3), \quad X_{4}=\frac{1}{2}\left(P_{0}+P_{4}\right) .
\end{gathered}
$$

Nonconjugate subalgebras of the Lie algebra of the group $P(1,4)$ have been described in the papers $[3,4,17]$.

The Lie algebra of the extended Galilei group $\widetilde{G}(1,3)$ is generated by the following bases elements:

$$
L_{1}, \quad L_{2}, \quad L_{3}, \quad P_{1}, \quad P_{2}, \quad P_{3}, \quad X_{0}, \quad X_{1}, \quad X_{2}, \quad X_{3}, \quad X_{4} .
$$

The classification of all nonconjugate subalgebras of the Lie algebra of the group $P(1,4)$ of dimensions $\leqslant 3$ was performed in [8].

3. ON CLASSIFICATION OF SYMMETRY REDUCTIONS USING

TWO-DIMENSIONAL NONCONJUGATE SUBALGEBRAS OF THE LIE Algebra of the Poincaré group $P(1,4)$

In this section, we consider the homogeneous Monge-Ampère equation in the space $M(1,3) \times R(u)$ :

$$
\operatorname{det}\left(u_{\mu \nu}\right)=0
$$

where

$$
u=u(x), \quad x=\left(x_{0}, x_{1}, x_{2}, x_{3}\right) \in M(1,3), \quad u_{\mu \nu} \equiv \frac{\partial^{2} u}{\partial x_{\mu} \partial x_{\nu}},
$$

for $\mu, \nu=0,1,2,3$. Here, and in what follows, $M(1,3)$ is a four-dimensional Minkowski space, and $R(u)$ is a real number axis of the depended variable $u$.

In 1983, Fushchich and Serov [16] studied symmetry properties and constructed some classes of exact solutions for the multidimensional MongeAmpère equation. It follows from that paper that the Lie algebra of the symmetry group of the equation under consideration contains, as subalgebra, the Lie algebra of the group $P(1,4)$. As we mentioned before, the results of the classification of all the low-dimensional ( $\operatorname{dim} L \leqslant 3$ ) nonconjugate subalgebras of the Lie algebra of the group $P(1,4)$ could be found in [8]. We will present below some of the results obtained.

3.1. Lie algebras of type $2 A_{1}$. Taking into account some invariants of two-dimensional nonconjugate subalgebras, we constructed the ansatzes reducing the $(1+3)$-dimensional homogeneous Monge-Ampère equation to identities. 
1) $\left\langle L_{3}-P_{3}\right\rangle \oplus\left\langle X_{4}\right\rangle$.

The first step is to construct a functional basis of the invariants for this subalgebra. In order to realize it we have solved the following system of PDEs:

$$
\left\{\begin{array}{l}
\left(L_{3}-P_{3}\right) \omega\left(x_{0}, x_{1}, x_{2}, x_{3}, u\right)=0, \\
\left(X_{4}\right) \omega\left(x_{0}, x_{1}, x_{2}, x_{3}, u\right)=0 .
\end{array}\right.
$$

The solutions of this system are

$$
\begin{aligned}
& \omega_{1}=\left(x_{1}^{2}+x_{2}^{2}\right)^{1 / 2}, \\
& \omega_{2}=\arctan \frac{x_{1}}{x_{2}}+\frac{x_{3}}{x_{0}+u}, \\
& \omega_{3}=x_{0}+u .
\end{aligned}
$$

From these invariants we construct an ansatz as follows

$$
x_{0}+u=\varphi\left(\omega_{1}, \omega_{2}\right), \quad \omega_{1}=\left(x_{1}^{2}+x_{2}^{2}\right)^{1 / 2}, \quad \omega_{2}=\arctan \frac{x_{1}}{x_{2}}+\frac{x_{3}}{x_{0}+u} .
$$

The second step is to calculate the second order derivatives $u_{\mu \nu}(\mu, \nu=$ $0,1,2,3)$. The third step is to substitute the derivatives into the equation under investigation $((1+3)$-dimensional homogeneous Monge-Ampère equation). This substitution reduces the equation under investigation to the identity of the form $0=0$. It means that the ansatz is a solution of the following $(1+3)$-dimensional homogeneous Monge-Ampère equation:

$$
x_{0}+u=\varphi\left(\sqrt{x_{1}^{2}+x_{2}^{2}}, \arctan \frac{x_{1}}{x_{2}}+\frac{x_{3}}{x_{0}+u}\right),
$$

where $\varphi$ is an arbitrary smooth function.

Since the method is the same for all of the subalgebras, we will omit the interim details and provide with the final results only.

2) $\left\langle L_{3}+\lambda G, \lambda>0\right\rangle \oplus\left\langle X_{3}\right\rangle$.

In this case we have the following ansatz:

$$
\begin{gathered}
\left(x_{1}^{2}+x_{2}^{2}\right)^{1 / 2}=\varphi\left(\omega_{1}, \omega_{2}\right), \\
\omega_{1}=\left(x_{0}^{2}-u^{2}\right)^{1 / 2}, \quad \omega_{2}=\ln \left(x_{0}+u\right)+\lambda \arctan \frac{x_{1}}{x_{2}},
\end{gathered}
$$

and the solution of the $(1+3)$-dimensional homogeneous Monge-Ampère equation has the form

$$
\left(x_{1}^{2}+x_{2}^{2}\right)^{1 / 2}=\varphi\left(x_{0}^{2}-u^{2}, \ln \left(x_{0}+u\right)+\lambda \arctan \frac{x_{1}}{x_{2}}\right),
$$

where $\varphi$ is an arbitrary smooth function. 
3) $\langle G\rangle \oplus\left\langle X_{1}\right\rangle$.

The ansatz is

$$
\left(x_{0}^{2}-u^{2}\right)^{1 / 2}=\varphi\left(\omega_{1}, \omega_{2}\right), \quad \omega_{1}=x_{2}, \quad \omega_{2}=x_{3},
$$

and the solution of the $(1+3)$-dimensional homogeneous Monge-Ampère equation has the form

$$
\left(x_{0}^{2}-u^{2}\right)^{1 / 2}=\varphi\left(x_{2}, x_{3}\right),
$$

where $\varphi$ is an arbitrary smooth function.

4) $\left\langle G+\alpha X_{2}, \alpha>0\right\rangle \oplus\left\langle X_{1}\right\rangle$.

The ansatz is

$$
x_{3}=\varphi\left(\omega_{1}, \omega_{2}\right), \quad \omega_{1}=\left(x_{0}^{2}-u^{2}\right)^{1 / 2}, \quad \omega_{2}=x_{2}-\alpha \ln \left(x_{0}+u\right),
$$

and the solution of the $(1+3)$-dimensional homogeneous Monge-Ampère equation has the form

$$
x_{3}=\varphi\left(x_{0}^{2}-u^{2}, x_{2}-\alpha \ln \left(x_{0}+u\right)\right),
$$

where $\varphi$ is an arbitrary smooth function.

5) $\left\langle L_{3}+\frac{1}{2}\left(P_{3}+C_{3}\right)\right\rangle \oplus\left\langle X_{0}+X_{4}\right\rangle$.

The ansatz is

$$
\begin{gathered}
\arctan \frac{x_{1}}{x_{2}}-\arctan \frac{x_{3}}{u}=\varphi\left(\omega_{1}, \omega_{2}\right), \\
\omega_{1}=\left(x_{1}^{2}+x_{2}^{2}\right)^{1 / 2}, \quad \omega_{2}=\left(u^{2}+x_{3}^{2}\right)^{1 / 2},
\end{gathered}
$$

and the solution of the $(1+3)$-dimensional homogeneous Monge-Ampère equation has the form

$$
\arctan \frac{x_{1}}{x_{2}}-\arctan \frac{x_{3}}{u}=\varphi\left(\sqrt{x_{1}^{2}+x_{2}^{2}}, \sqrt{u^{2}+x_{3}^{2}}\right),
$$

where $\varphi$ is an arbitrary smooth function.

6) $\left\langle L_{3}+\frac{\lambda}{2}\left(P_{3}+C_{3}\right), 0<\lambda<1\right\rangle \oplus\left\langle X_{0}+X_{4}\right\rangle$.

The ansatz is

$$
\begin{gathered}
\lambda \arctan \frac{x_{1}}{x_{2}}-\arctan \frac{x_{3}}{u}=\varphi\left(\omega_{1}, \omega_{2}\right), \\
\omega_{1}=\left(x_{1}^{2}+x_{2}^{2}\right)^{1 / 2}, \quad \omega_{2}=\left(u^{2}+x_{3}^{2}\right)^{1 / 2},
\end{gathered}
$$

and the solution of the $(1+3)$-dimensional homogeneous Monge-Ampère equation has the form

$$
\lambda \arctan \frac{x_{1}}{x_{2}}-\arctan \frac{x_{3}}{u}=\varphi\left(\sqrt{x_{1}^{2}+x_{2}^{2}}, \sqrt{u^{2}+x_{3}^{2}}\right),
$$

where $\varphi$ is an arbitrary smooth function. 
7) $\left\langle L_{3}+\alpha\left(X_{0}+X_{4}\right), \alpha>0\right\rangle \oplus\left\langle X_{4}\right\rangle$.

The ansatz is

$$
\begin{gathered}
x_{0}+u-\alpha \arctan \frac{x_{1}}{x_{2}}=\varphi\left(\omega_{1}, \omega_{2}\right), \\
\omega_{1}=x_{3}, \quad \omega_{2}=\left(x_{1}^{2}+x_{2}^{2}\right)^{1 / 2},
\end{gathered}
$$

and the solution of the $(1+3)$-dimensional homogeneous Monge-Ampère equation has the form

$$
u=\varphi\left(x_{3}, \sqrt{x_{1}^{2}+x_{2}^{2}}\right)+\alpha \arctan \frac{x_{1}}{x_{2}}-x_{0},
$$

where $\varphi$ is an arbitrary smooth function.

8) $\left\langle L_{3}+\alpha X_{3}, \alpha>0\right\rangle \oplus\left\langle X_{0}+X_{4}\right\rangle$.

The ansatz is

$$
x_{3}+\alpha \arctan \frac{x_{1}}{x_{2}}=\varphi\left(\omega_{1}, \omega_{2}\right), \quad \omega_{1}=u, \quad \omega_{2}=\left(x_{1}^{2}+x_{2}^{2}\right)^{1 / 2},
$$

and the solution of the $(1+3)$-dimensional homogeneous Monge-Ampère equation has the form

$$
x_{3}+\alpha \arctan \frac{x_{1}}{x_{2}}=\varphi\left(u, \sqrt{x_{1}^{2}+x_{2}^{2}}\right),
$$

where $\varphi$ is an arbitrary smooth function.

9) $\left\langle L_{3}+2 X_{4}\right\rangle \oplus\left\langle X_{3}\right\rangle$.

The ansatz is

$$
\begin{gathered}
x_{0}-u+2 \arctan \frac{x_{2}}{x_{1}}=\varphi\left(\omega_{1}, \omega_{2}\right), \\
\omega_{1}=x_{0}+u, \quad \omega_{2}=\left(x_{1}^{2}+x_{2}^{2}\right)^{1 / 2},
\end{gathered}
$$

and the solution of the $(1+3)$-dimensional homogeneous Monge-Ampère equation has the form

$$
x_{0}-u+2 \arctan \frac{x_{2}}{x_{1}}=\varphi\left(x_{0}+u, \sqrt{x_{1}^{2}+x_{2}^{2}}\right),
$$

where $\varphi$ is an arbitrary smooth function.

10) $\left\langle L_{3}-P_{3}+2 \alpha X_{0}, \alpha \neq 0\right\rangle \oplus\left\langle X_{4}\right\rangle$.

The ansatz is

$$
\begin{gathered}
x_{0}+u-2 \alpha \arctan \frac{x_{1}}{x_{2}}=\varphi\left(\omega_{1}, \omega_{2}\right), \\
\omega_{1}=\left(x_{1}^{2}+x_{2}^{2}\right)^{1 / 2}, \quad \omega_{2}=\left(x_{0}+u\right)^{2}+4 \alpha x_{3},
\end{gathered}
$$


and the solution of the $(1+3)$-dimensional homogeneous Monge-Ampère equation has the form

$$
u=\varphi\left(\sqrt{x_{1}^{2}+x_{2}^{2}},\left(x_{0}+u\right)^{2}+4 \alpha x_{3}\right)+2 \alpha \arctan \frac{x_{1}}{x_{2}}-x_{0},
$$

where $\varphi$ is an arbitrary smooth function.

11) $\left\langle P_{3}\right\rangle \oplus\left\langle X_{1}\right\rangle$.

The ansatz is

$$
\left(x_{0}^{2}-x_{3}^{2}-u^{2}\right)^{1 / 2}=\varphi\left(\omega_{1}, \omega_{2}\right), \quad \omega_{1}=x_{2}, \quad \omega_{2}=x_{0}+u,
$$

and the solution of the $(1+3)$-dimensional homogeneous Monge-Ampère equation has the form

$$
\left(x_{0}^{2}-x_{3}^{2}-u^{2}\right)^{1 / 2}=\varphi\left(x_{2}, x_{0}+u\right),
$$

where $\varphi$ is an arbitrary smooth function.

12) $\left\langle P_{3}-X_{2}\right\rangle \oplus\left\langle X_{1}\right\rangle$.

The ansatz is

$$
x_{2}-\frac{x_{3}}{x_{0}+u}=\varphi\left(\omega_{1}, \omega_{2}\right), \quad \omega_{1}=x_{0}+u, \quad \omega_{2}=\left(x_{0}^{2}-x_{3}^{2}-u^{2}\right)^{1 / 2},
$$

and the solution of the $(1+3)$-dimensional homogeneous Monge-Ampère equation has the form

$$
x_{2}-\frac{x_{3}}{x_{0}+u}=\varphi\left(x_{0}+u, \sqrt{x_{0}^{2}-x_{3}^{2}-u^{2}}\right),
$$

where $\varphi$ is an arbitrary smooth function.

13) $\left\langle P_{3}-2 X_{0}\right\rangle \oplus\left\langle X_{4}\right\rangle$.

The ansatz is

$$
\left(x_{0}+u\right)^{2}+4 x_{3}=\varphi\left(\omega_{1}, \omega_{2}\right), \quad \omega_{1}=x_{1}, \quad \omega_{2}=x_{2},
$$

and the solution of the $(1+3)$-dimensional homogeneous Monge-Ampère equation has the form

$$
\left(x_{0}+u\right)^{2}+4 x_{3}=\varphi\left(x_{1}, x_{2}\right),
$$

where $\varphi$ is an arbitrary smooth function.

14) $\left\langle P_{3}-2 X_{0}\right\rangle \oplus\left\langle X_{1}\right\rangle$.

The ansatz is

$$
\begin{gathered}
\frac{1}{6}\left(x_{0}+u\right)^{3}+x_{3}\left(x_{0}+u\right)+x_{0}-u=\varphi\left(\omega_{1}, \omega_{2}\right), \\
\omega_{1}=x_{2}, \quad \omega_{2}=\left(x_{0}+u\right)^{2}+4 x_{3},
\end{gathered}
$$


and the solution of the $(1+3)$-dimensional homogeneous Monge-Ampère equation has the form

$$
\frac{1}{6}\left(x_{0}+u\right)^{3}+x_{3}\left(x_{0}+u\right)+x_{0}-u=\varphi\left(x_{2},\left(x_{0}+u\right)^{2}+4 x_{3}\right),
$$

where $\varphi$ is an arbitrary smooth function.

15) $\left\langle L_{3}\right\rangle \oplus\left\langle X_{4}\right\rangle$.

The ansatz is

$$
\left(x_{1}^{2}+x_{2}^{2}\right)^{1 / 2}=\varphi\left(\omega_{1}, \omega_{2}\right), \quad \omega_{1}=x_{0}+u, \quad \omega_{2}=x_{3},
$$

and the solution of the $(1+3)$-dimensional homogeneous Monge-Ampère equation has the form

$$
\left(x_{1}^{2}+x_{2}^{2}\right)^{1 / 2}=\varphi\left(x_{0}+u, x_{3}\right),
$$

where $\varphi$ is an arbitrary smooth function.

16) $\left\langle L_{3}+\alpha X_{3}, \alpha>0\right\rangle \oplus\left\langle X_{4}\right\rangle$.

The ansatz is

$$
x_{3}+\alpha \arctan \frac{x_{1}}{x_{2}}=\varphi\left(\omega_{1}, \omega_{2}\right), \quad \omega_{1}=x_{0}+u, \quad \omega_{2}=\left(x_{1}^{2}+x_{2}^{2}\right)^{1 / 2},
$$

and the solution of the $(1+3)$-dimensional homogeneous Monge-Ampère equation has the form

$$
x_{3}+\alpha \arctan \frac{x_{1}}{x_{2}}=\varphi\left(x_{0}+u,\left(x_{1}^{2}+x_{2}^{2}\right)^{1 / 2}\right),
$$

where $\varphi$ is an arbitrary smooth function.

17) $\left\langle P_{3}-X_{1}\right\rangle \oplus\left\langle X_{4}\right\rangle$.

The ansatz is

$$
x_{1}-\frac{x_{3}}{x_{0}+u}=\varphi\left(\omega_{1}, \omega_{2}\right), \quad \omega_{1}=x_{2}, \quad \omega_{2}=x_{0}+u,
$$

and the solution of the $(1+3)$-dimensional homogeneous Monge-Ampère equation has the form

$$
x_{1}-\frac{x_{3}}{x_{0}+u}=\varphi\left(x_{2}, x_{0}+u\right),
$$

where $\varphi$ is an arbitrary smooth function.

3.2. Lie algebras of type $A_{2}$. Taking into account some invariants of twodimensional nonconjugate subalgebras, we constructed the ansatzes, which reduced the $(1+3)$-dimensional homogeneous Monge-Ampère equation to identities. 
1) $\left\langle-G-\frac{1}{\lambda} L_{3}, X_{4}, \lambda>0\right\rangle$.

The ansatz is

$\ln \left(x_{0}+u\right)+\lambda \arctan \frac{x_{1}}{x_{2}}=\varphi\left(\omega_{1}, \omega_{2}\right), \quad \omega_{1}=x_{3}, \quad \omega_{2}=\left(x_{1}^{2}+x_{2}^{2}\right)^{1 / 2}$,

and the solution of the $(1+3)$-dimensional homogeneous Monge-Ampère equation has the form

$$
\ln \left(x_{0}+u\right)+\lambda \arctan \frac{x_{1}}{x_{2}}=\varphi\left(x_{3},\left(x_{1}^{2}+x_{2}^{2}\right)^{1 / 2}\right),
$$

where $\varphi$ is an arbitrary smooth function.

2) $\left\langle-G-\alpha X_{1}, X_{4}, \alpha>0\right\rangle$.

The ansatz is

$$
x_{1}-\alpha \ln \left(x_{0}+u\right)=\varphi\left(\omega_{1}, \omega_{2}\right), \quad \omega_{1}=x_{2}, \quad \omega_{2}=x_{3},
$$

and the solution of the $(1+3)$-dimensional homogeneous Monge-Ampère equation has the form

$$
x_{1}-\alpha \ln \left(x_{0}+u\right)=\varphi\left(x_{2}, x_{3}\right),
$$

where $\varphi$ is an arbitrary smooth function.

$3)\left\langle-\frac{1}{\lambda}\left(L_{3}+\lambda G+\alpha X_{3}\right), X_{4}, \alpha>0, \lambda>0\right\rangle$.

The ansatz is

$$
\begin{gathered}
\ln \left(x_{0}+u\right)+\lambda \arctan \frac{x_{1}}{x_{2}}=\varphi\left(\omega_{1}, \omega_{2}\right), \\
\omega_{1}=\left(x_{1}^{2}+x_{2}^{2}\right)^{1 / 2}, \quad \omega_{2}=x_{3}+\alpha \arctan \frac{x_{1}}{x_{2}},
\end{gathered}
$$

and the solution of the $(1+3)$-dimensional homogeneous Monge-Ampère equation has the form

$$
\ln \left(x_{0}+u\right)+\lambda \arctan \frac{x_{1}}{x_{2}}=\varphi\left(\left(x_{1}^{2}+x_{2}^{2}\right)^{1 / 2}, x_{3}+\alpha \arctan \frac{x_{1}}{x_{2}}\right),
$$

where $\varphi$ is an arbitrary smooth function.

\section{Conclusions}

We study the relationship between the structural properties of the lowdimensional ( $\operatorname{dim} L \leqslant 3$ ) nonconjugate subalgebras of the same rank of the Lie algebra of the Poincaré group $P(1,4)$ and the properties of the reduced equations for the $(1+3)$-dimensional homogeneous Monge-Ampère equation.

At the present time, the relationship has been investigated between the structural properties of the two-dimensional nonconjugate subalgebras of the same rank of the Lie algebra of the group $P(1,4)$ and the properties of reduced equations for the $(1+3)$-dimensional homogeneous Monge-Ampère equation. We have obtained the following types of the reduced equations: 
- identities,

- partial differential equations.

In this paper, we have presented some of our results, which were obtained on the way of symmetry reduction of the $(1+3)$-dimensional homogeneous Monge-Ampère equation to identities. More detailed: we provided some classes of the invariant solutions (with arbitrary smooth functions) for the equation under investigation. Those classes were divided into two subclasses: subclasses invariant under nonconjugate subalgebras of the Lie algebra of the group $P(1,4)$ of the type $2 A_{1}$ and subclasses invariant under nonconjugate subalgebras of the Lie algebra of the group $P(1,4)$ of the type $A_{2}$. To do that we have used the classification of all nonconjugate subalgebras of the Lie algebra of the group $P(1,4)$ of dimensions $\leqslant 3$ which was performed in [8].

From the results obtained it follows that the reductions to identities can be obtained by using some subalgebras of the following types: $2 A_{1}$ and $A_{2}$.

It should be noted that the ansatzes (non-singular manifolds in the space $M(1,3) \times R(u)$, invariant with respect to the corresponding subalgebras) are classes of the invariant solutions (with arbitrary smooth functions) of the $(1+3)$-dimensional homogeneous Monge-Ampère equation.

\section{REFERENCES}

[1] S. Y. Cheng. On the real and complex Monge-Ampère equation and its geometric applications. In Proceedings of the International Congress of Mathematicians, Vol. 1, 2 (Warsaw, 1983), pages 533-539. PWN, Warsaw, 1984.

[2] M. J. P. Cullen, R. J. Douglas. Applications of the Monge-Ampère equation and Monge transport problem to meteorology and oceanography, volume 226 of Contemp. Math. Amer. Math. Soc., Providence, RI, 1999, doi: 10.1090/conm/226/03234.

[3] V. M. Fedorchuk. Splitting subalgebras of the Lie algebra of the generalized Poincaré group $p(1,4)$. Ukr. Mat. Zh., 31:717-722, 1979.

[4] V. M. Fedorchuk. Nonsplitting subalgebras of the Lie algebra of the generalized Poincaré group $p(1,4)$. Ukr. Mat. Zh., 33:696-700, 1981.

[5] V. M. Fedorchuk. Symmetry reduction and exact solutions of the Euler-Lagrange-BornInfeld, multidimensional Monge-Ampere and eikonal equations. J. Nonlinear Math. Phys., 2(3-4):329-333, 1995, doi: 10.2991/jnmp.1995.2.3-4.13. Symmetry in nonlinear mathematical physics, Vol. 1 (Kiev, 1995).

[6] V. M. Fedorchuk. Symmetry reduction and some exact solutions of a nonlinear five-dimensional wave equation. Ukraïn. Mat. Zh., 48(4):573-576, 1996, doi: $10.1007 / \mathrm{BF} 02390625$.

[7] V. M. Fedorchuk, I. M. Fedorchuk, O. S. Leibov. Reduction of the Born-Infeld, the Monge-Ampère and the eikonal equation to linear equations. Dokl. Akad. Nauk Ukrainy, (11):24-27, 173, 1991.

[8] V. M. Fedorchuk, V. I. Fedorchuk. On classification of the low-dimensional nonconjugate subalgebras of the Lie algebra of the Poincare group $P(1,4)$, volume 3 . Kyiv, Ukraine: Institut of Mathematics of NAS of Ukraine, 2006. 
[9] V. M. Fedorchuk, V. I. Fedorchuk. On classification of symmetry reductions for the eikonal equation. Symmetry, 8(6):Art. 51, 32, 2016, doi: 10.3390/sym8060051.

[10] V. M. Fedorchuk, V. I. Fedorchuk. On classification of symmetry reductions for partial differential equations. Collection of the works dedicated to 80th of anniversary of B. J. Ptashnyk, pages 241-255, 2017.

[11] V. M. Fedorchuk, V. I. Fedorchuk. Classification of Symmetry Reductions for the Eikonal Equation. Lviv: Pidstryhach Institute for Applied Problems of Mechanics and Mathematics of National Academy of Sciences of Ukraine, 2018.

[12] V. M. Fedorchuk, V. I. Fedorchuk. On symmetry reduction of the Euler-Lagrange-BornInfeld equation to linear odes. Symmetry and Integrability of Equations of Mathematical Physics. Collection of Works of Institute of Mathematics, 16:193-202, 2019.

[13] V. M. Fedorchuk, V. I. Fedorchuk. On the classification of symmetry reduction and invariant solutions for the Euler-Lagrange-Born-Infeld equation. Ukr. J. Phys., 64:11031107, 2019, doi: 10.15407/ujpe64.12.1103.

[14] V. M. Fedorchuk, V. I. Fedorchuk. On the classification of symmetry reductions for the $(1+3)$-dimensional Monge-Ampère equation. Mathematical methods and physicomechanical fields, 63(2):7-16, 2020.

[15] Alessio Figalli. The Monge-Ampère equation and its applications. Zurich Lectures in Advanced Mathematics. European Mathematical Society (EMS), Zürich, 2017, doi: $10.4171 / 170$.

[16] V. I. Fushchich, N. I. Serov. Symmetry and some exact solutions of the multidimensional Monge-Ampère equation. Dokl. Akad. Nauk SSSR, 273(3):543-546, 1983.

[17] W. I. Fushchich, A. F. Barannik, L. F. Barannik, V. M. Fedorchuk. Continuous subgroups of the Poincaré group $P(1,4)$. J. Phys. A, 18(15):2893-2899, 1985, http: //stacks.iop.org/0305-4470/18/2893.

[18] W. I. Fushchich, A. G. Nikitin. Reduction of the representations of the generalized Poincaré algebra by the galilei algebra. J. Phys. A: Math. and Gen, 13:2319-2330, 1980.

[19] A. M. Grundland, A. Hariton. Algebraic aspects of the supersymmetric minimal surface equation. Symmetry, 9(12):318, 2017, doi: 10.3390/sym9120318.

[20] A. M. Grundland, J. Harnad, P. Winternitz. Symmetry reduction for nonlinear relativistically invariant equations. J. Math. Phys., 25(4):791-806, 1984, doi: $10.1063 / 1.526224$.

[21] F. Jiang, N. S. Trudinger. On the second boundary value problem for Monge-Ampère type equations and geometric optics. Arch. Ration. Mech. Anal., 229(2):547-567, 2018, doi: $10.1007 / \mathrm{s} 00205-018-1222-8$.

[22] S. V. Khabirov. Application of contact transformations of the inhomogeneous Monge-Ampère equation in one-dimensional gas dynamics. Dokl. Akad. Nauk SSSR, 310(2):333-336, 1990.

[23] S. Kołodziej, N. C. Nguyen. Continuous solutions to Monge-Ampère equations on Hermitian manifolds for measures dominated by capacity. Calc. Var. Partial Differential Equations, 60(3):Paper No. 93, 18, 2021, doi: 10.1007/s00526-021-01944-4.

[24] A. Kushner, V. Lychagin, J. Slovák. Lectures on geometry of Monge-Ampère equations with Maple. Tutor. Sch. Workshops Math. Sci. Birkhäuser/Springer, Cham, 2019.

[25] Nam Q. Le. Global Hölder estimates for 2D linearized Monge-Ampère equations with right-hand side in divergence form. J. Math. Anal. Appl., 485(2):123865, 13, 2020, doi: $10.1016 /$ j.jmaa.2020.123865. 
[26] M. Lewicka, L. Mahadevan, M. R. Pakzad. The Monge-Ampère constraint: matching of isometries, density and regularity, and elastic theories of shallow shells. Ann. Inst. H. Poincaré Anal. Non Linéaire, 34(1):45-67, 2017, doi: 10.1016/j.anihpc.2015.08.005.

[27] S. Lie. Zur allgemeinen Theorie der partiellen Differentialgleichungen beliebiger Ordnung. Berichte Sächs. Ges., 2:53-128, 1895.

[28] A. G. Nikitin, O. Kuriksha. Invariant solutions for equations of axion electrodynamics. Commun. Nonlinear Sci. Numer. Simul., 17(12):4585-4601, 2012, doi: $10.1016 /$ j.cnsns.2012.04.009.

[29] Francesco Oliveri. Lie symmetries of differential equations: classical results and recent contributions. Symmetry, 2(2):658-706, 2010, doi: 10.3390/sym2020658.

[30] Peter J. Olver. Applications of Lie groups to differential equations, volume 107 of Graduate Texts in Mathematics. Springer-Verlag, New York, 1986, doi: 10.1007/978-14684-0274-2.

[31] L. V. Ovsiannikov. Group analysis of differential equations. Moscow: Nauka, 1978.

[32] J. Patera, P. Winternitz, H. Zassenhaus. Continuous subgroups of the fundamental groups of physics. I. General method and the Poincaré group. J. Math. Phys., 16:15971614, 1975, doi: 10.1063/1.522729.

[33] A. V. Pogorelov. Multidimensional Minkovski problem. Izdat. "Nauka", Moscow, 1975 (in Russian).

[34] A. V. Pogorelov. Multidimensional Monge-Ampère equation $\operatorname{det}\left\|z_{i j}\right\|=$ $\phi\left(z_{1}, \cdots, z_{n}, z, x_{1}, \cdots, x_{n}\right)$. "Nauka", Moscow, 1988 (in Russian).

[35] M. B. Sheftel. Nonlocal symmetry of CMA generates ASD Ricci-flat metric with no Killing vectors. J. Math. Phys., 62(1):Paper No. 013504, 17, 2021, doi: $10.1063 / 5.0022021$.

[36] Ł. T. Stȩpień. On some exact solutions of heavenly equations in four dimensions. AIP Advances, 10(6):065105, 2020, doi: 10.1063/1.5144327.

[37] C. Udrişte, N. Bîlă. Symmetry Lie group of the Monge-Ampère equation. Balkan J. Geom. Appl., 3(2):121-134, 1998.

[38] C. Udrişte, N. Bîlă. Symmetry group of Ţiţeica surfaces pde. Balkan J. Geom. Appl., 4(2):123-140, 1999.

[39] Xu-Jia Wang. Monge-Ampère equation and optimal transportation. In Proceedings of the Sixth International Congress of Chinese Mathematicians. Vol. I, volume 36 of Adv. Lect. Math. (ALM), pages 153-172. Int. Press, Somerville, MA, 2017.

[40] Shing-Tung Yau, Steve Nadis. The shape of a life. Yale University Press, New Haven, CT, 2019, doi: 10.2307/j.ctvbnm3qt.

\section{Vasyl Fedorchuk}

Pidstryhach Institute for Applied Problems of Mechanics and Mathematics of NAS of Ukraine, 79060, 3-B Naukova St., Lviv, Ukraine

Email: vasfed@gmail.com

\section{Volodymyr Fedorchuk}

Pidstryhach Institute for Applied Problems of Mechanics and Mathematics of NAS of Ukraine, 79060, 3-B Naukova St., Lviv, Ukraine

Email: volfed@gmail.com 